\title{
CONTENIDO DE METALES PESADOS EN CRASSOSTREA ANGULATA, DICENTRARCHUS LABRAX Y MUGIL AURATUS DE LOS ESTUARIOS DE LOS RIOS GUADALQUIVIR Y BARBATE
}

\author{
R. Cordón; F. Cabrera; E. Díaz y P. de Arambarri \\ Centro de Edafologia y Biología Aplicada del Cuarto (C.S.I.C.). Apartado 1.052. 41080 Sevilla.
}

Palabras clave: Heavy metals, pollution, estuaries, Crassostrea angulata, Dicentrarchus labrax, Mugil auratus.

\author{
ABSTRACT \\ HEAVY METALS CONTENTS OF CRASSOSTREA ANGULATA, DICENTRARCHUS LABRAX \\ AND MUGIL AURATUS FROM THE ESTUARIES OF GUADALQUIVIR \\ AND BARBATE RIVERS.
}

\begin{abstract}
Heavy metal contents ( $\mathrm{Fe}, \mathrm{Cu}, \mathrm{Mn}$ and $\mathrm{Zn}$ ) of oyster (Crassostrea angulata), european sea bass (Dicentrarchus labras) and grey mullet (Mugil auratus) from Guadalquivir and Barbate estuanes were compared. Every comparison was made between species of the same range of weight.

Edible tissue of oysters from the Guadalquivirestuary had average concentrations of $\mathrm{Cu}$ and $\mathrm{Zn} 5.5$ and 2.2 times higher respectively than those from Barbate. Copper concentrations of oysters from Guadalquivir estuary were always higher than the recommended permissible concentration for human consumption $(1.000 \mu \mathrm{g} \mathrm{Cu} / \mathrm{g} \mathrm{d.w}$.).

Livers ofeuropean sea bass from Guadalquivirestuary had mean $\mathrm{Cu}$ concentration 3.0 times higher than those from Barbate.

Mean concentrations of $\mathrm{Fe}, \mathrm{Cu}$ and $\mathrm{Zn}$ of grey mullet livers also were 1.7, 22.7 and 1.6 times higher respectively in specimens from Guadalquivirriver.

Concentrationsfor all metals is the two fish species studied were higher in livers than in dorsal muscle tissues. Copper accumulation in liver ofboth sea bass and grey mullet were higher in specimens from Guadalquivirestuary.
\end{abstract}

\section{INTRODUCCION}

En el litoral suratlántico español existe una franja costera, comprendida entre la ría de Huelva y la Punta de Rota, en la cual la contaminación de moluscos por metales pesados puede ser elevada, destacando el caso del cobre cuyas concentraciones en ostiones (Crassostrea angulata) superan en mucho los niveles admitidos para el consumo humano $(150 \mu \mathrm{g} / \mathrm{g}$ peso húmedo). En el mismo litoral, entre Rota y la desembocadura del río Barbate, los ostiones tienen concentraciones normales de este elemento (Establier, 1969; Establier y Pascual, 1974).

Se especula con la posibilidad de que la contaminación existente entre Huelva y Rota pueda deberse a la incidencia del río Guadalquivir y de la ría de Huelva, provocada por la deriva litoral de la zona.

A excepción de algunas especies de moluscos (ostión, lapa y almeja), muy estudiadas desde el punto

Limnética 3: 159-164 (1987)

(C) Asociación Española de Limnología, Madrid. Spain de vista de la contaminación metálica (Establier, 1977), se ha prestado escasa atención a especies piscícolas. Por ello, en el presente trabajo se han estudiado tres especies de elevado interés comercial en la zona, pertenecientes a distintos niveles troficos: ostión (Crassostrea angulata), filtrador; lisa (Mugilauratus), detritivoro; y lubina (Dicentrarchus labrax), depredador. Estas especies se capturaron en los estuarios de los rios Guadalquivir y Barbate, con objeto de establecer comparaciones entre los dos ríos, ya que el Guadalquivir es receptor y portador de importantes vertidos urbanos, mineros, agrícolas, etc., mientras que el Barbate solo está afectado por vertidos agrícolas y urbanos poco importantes.

\section{MATERIAL Y METODOS}

Las muestras fueron recogidas en los dos estuarios estudiados por personal del Plan de Explotación Ma- 
n

$\mu \mathrm{g} / \mathrm{g}$ peso seco

$\mathrm{Cu}$

$\mu \mathrm{g} / \mathrm{g}$ peso seco

$\mathrm{Mn}$

$\mu \mathrm{g} / \mathrm{g}$ peso seco

$\mathrm{Zn}$

$\mu \mathrm{g} / \mathrm{g}$ peso seco
Máx.

Mín.

$\overline{\mathrm{X}}$

S

Máx.

Min.

$\overline{\mathrm{X}}$

S

Máx

Mín.

$\mathrm{X}$

S

Máx.

Mín.

$\overline{\mathrm{x}}$

$\mathrm{S}$

\section{Ostiones}

Barbate

17

1.600
257
681 (a)
384,0

1.520

302

$545(\mathrm{~b})$ * * *

303,1

76,1

17,7 (c)

16,42

8.333

2.895

4.447 (d)

$1.379,2$ $\underset{41}{\text { Guadalquivir }}$

2.082

342

741 (a)

339,6

5.952

1.034

$2.979(\mathrm{~b})$ * * *

$1.061,9$

85

19,5

44,3 (c)

13,10

20.742

2.422

$9.729(\mathrm{~d})$

$3.905,5$

Las letras iguales entre paréntesis, muestran los conjuntos de datos comparados entre sí por el criterio «t» de Student.

Grado de significación: ${ }^{* *} \mathrm{p}<0,001$

Tabla 1.- Ostiones (Crassostreaangulata). Número de individuos (n), valores máximos y mínimos, valores medios ( $\bar{x}$ ), y desviaciones típicas (s) de las concentraciones de hierro, cobre, manganeso y cinc en individuos con peso $<10 \mathrm{~g}$.

Oysters (Crassostreaangulata). Number of specimens(n), maximum (Max.), minimum (Min.), mean ( $\overline{\mathrm{x}}$ ) values and standard deviations (s) of the concentrations of $\mathrm{Fe}, \mathrm{Cu}, \mathrm{Mn}$ and $\mathrm{Zn}$ in specimens weighing less than $10 \mathrm{~g}$.

risquera y Cultivos Marinos de la Región Suratlántica (P.E.M.A.R.E.S., Cádiz), en el otoño de 1983 y la primavera de 1984. En el primer muestreo se capturaron en el estuario de Barbate 20 ejemplares de ostión, 12 de lubina y 13 de lisa, y 22, 11 y 10 respectivamente en el del Guadalquivir. En el segundo muestreo se capturaron en el del Barbate 17 ostiones, 16 lubinas y 19 lisas, y 20, 8 y 17 respectivamente en el del Guadalquivir.

Los ostiones se midieron, extrajeron de las valvas y pesaron. Los ejemplares de lubina y lisa se midieron, pesaron y diseccionaron para extraer los hígados y musculatura dorsal. Los ostiones desprovistos de valvas, los hígados y muestras de $5 \mathrm{~g}$. de musculatura dorsal de lubinas y lisas se secaron a $90^{\circ} \mathrm{C}$ durante 48 h. para la determinación de la pérdida de peso. Las muestras desecadas se disolvieron por los métodos recomendados en la bibliografía (Ritter et al., 1978; Katz et al., 1981; Lytle y Lytle 1982), y posteriormente se determinaron en ellas, por espectroscopía de absorción atómica, las concentraciones de hierro, cobre, manganeso y cinc.

\section{RESULTADOS}

En ninguna de las dos zonas estudiadas se encontraron diferencias claras entre los contenidos de hierro, cobre, manganeso y cinc en los ostiones, lubinas y lisas capturados en otoño y primavera.

No se ha observado una tendencia general clara entre el peso total y las concentraciones de metales en los ostiones, ni en los hígados y musculatura de lubinas y lisas, aunque en la mayoría de los casos la concentración de metales tiende a disminuir conforme aumenta el peso del individuo. Habida cuenta de que los intervalos de peso en que se encontraban comprendidos los individuos de ambas zonas eran muy diferentes, se optó por comparar sólo aquellos individuos comprendidos en intervalos de peso comunes.

Comparando los ostiones comprendidos en el mismo intervalo de peso $(<10 \mathrm{~g})$ se observa que los valores medios de las concentraciones de hierro, cobre, manganeso y cinc son respectivamente 1,09, 5,47; 1,03 y 2,18 veces mayores en el estuario del Guadalquivir que en el del Barbate. Estas diferencias son sig- 


\begin{tabular}{|c|c|c|c|c|c|c|c|}
\hline & & Hígad & & & isculos & & $\begin{array}{l}\text { hígados/ } \\
\text { músculos }\end{array}$ \\
\hline & $\mathbf{n}$ & $\begin{array}{c}\text { Barbate } \\
27\end{array}$ & $\begin{array}{c}\text { Guadalquivir } \\
13\end{array}$ & $\begin{array}{c}\text { Barbate } \\
\quad 33\end{array}$ & $\begin{array}{c}\text { Guadalquivir } \\
\mathbf{1 4}\end{array}$ & Barbate & Guadalquivir \\
\hline $\mathrm{Fe}$ & Máx. & 2.664 & 1.949 & 32,9 & 35,7 & & \\
\hline$\mu \mathrm{g} / \mathrm{g}$ & Mín. & 206,3 & 410 & 6,2 & 9,2 & & \\
\hline peso seco & $\overline{\mathrm{x}}$ & 1.036 (a) & 919 (a) & 19,7 (e) & 23,2 (e) & 52,6 & 38,9 \\
\hline & $\mathrm{s}$ & 756.2 & 431,8 & 8,05 & 8,01 & & \\
\hline $\mathrm{Cu}$ & Máx. & 920,1 & 634 & 23,66 & 6,4 & & \\
\hline$\mu \mathrm{g} / \mathrm{g}$ & Mín. & 14,5 & 84,1 & 2,89 & 3,88 & & \\
\hline peso seco & $\bar{x}$ & $96,0(\mathrm{~b})^{* * *}$ & $291(\mathrm{~b})^{* *}$ & $8,15(\mathrm{f}) * * *$ & $4,84(\mathrm{f}){ }^{* * *}$ & 11,8 & 60,1 \\
\hline & $\mathrm{s}$ & 178,40 & 177,6 & 5,378 & 0,856 & & \\
\hline $\mathrm{Mn}$ & Máx. & 25,0 & 12,2 & 1,35 & 1,55 & & \\
\hline$\mu \mathrm{g} / \mathrm{g}$ & Mín. & 0,00 & 4,7 & 0,00 & 0,00 & & \\
\hline peso seco & $\overline{\mathbf{x}}$ & $10,3(c)$ & $9,5(\mathrm{c})$ & $0,82(\mathrm{~g})$ & 0,88 (g) & 12,6 & 10,8 \\
\hline & $\mathrm{s}$ & 7,45 & 2,28 & 0,557 & 0,440 & & \\
\hline $\mathrm{Zn}$ & Max. & 310,0 & 259 & 51,5 & 32,6 & & \\
\hline & Mín. & 97,2 & 116 & 12,7 & 18,6 & & \\
\hline peso seco & $x$ & $166(\mathrm{~d})$ & 164 (d) & $28,1(\mathrm{~h})^{*}$ & $23,5(\mathrm{~h})$ * & 5,9 & 7.0 \\
\hline & s & 53,7 & 39,8 & 7,73 & 3,66 & & \\
\hline
\end{tabular}

Las letras iguales entre paréntesis, muestran los conjuntos de datos comparados entre sí por el criterio «t» de Student.

Grupo de significación: $\begin{array}{rl}{ }^{*} p & <0,5 \\ * * & p<0,01 \\ * * * & p<0,001\end{array}$

Tabla 2.-(Dicentrarchus labrax). Concentraciones de metales en hígados y tejido de musculatura dorsal de individuos con peso $<$ 500 g. y relación de las concentraciones medias hígados/músculos. (n, numero de individuos; valores máximos y mínimos; $\overline{\mathbf{x}}$, valores medios; s, desviacionestípicas).

European sea bass (Dicentrarchus labrax). Metal concentrations in livers and dorsal muscle tissues of specimens weiphing less than $500 \mathrm{~g}$, and liver/muscle mean concentration ratios. (n, number of specimens; Max., maximum values; Min., minimum values; $\overline{\mathrm{x}}$, mean values; $\mathrm{s}$, standard deviations).

nificativas estadísticamente $(\mathrm{P}<0,001)$ en los casos del cobre y del cinc (Tabla 1$)$.

En la Tabla 2 se observa que los valores medios de las concentraciones de hierro, manganeso y cinc en los hígados de las lubinas comprendidas en el mismo intervalo de peso ( $\leqslant 500$ g.), son del mismo orden en ambas zonas de muestreo. En cambio, el valor medio de cobre en los hígados de los individuos del Guadalquivir es 3,03 veces superior $(P<0,01)$ al de los del Barbate. En cuanto a las concentraciones de metales en tejido de musculatura dorsal, sólo se han encontrado diferencias significativas en los valores medios de cobre y cinc $(\mathrm{P}<0,001 ; \mathrm{P}<0,5)$, que resultan ser respectivamente 1,7 y 1,2 veces mayores en los individuos procedentes del Barbate (Tabla 2).

Las concentraciones medias de hierro, cobre y cinc en hígados de lisas con peso total $\leqslant 700$ g. procedentes del estuario del Guadalquivir son respectivamente 1,$73 ; 22,67$ y 1,57 veces superiores a las correspondientes del Barbate $(\mathrm{P}<0,001)$, mientras que los valores medios de manganeso son muy similares en ambas zonas. Cabe destacar las altas concentraciones de hierro y cobre que alcanzan los hígados de algunos individuos de ambas zonas de muestreo $(3.180 \mu \mathrm{g}$ $\mathrm{Fe} / \mathrm{g}, 1.078 \mu \mathrm{g} \mathrm{Cu} / \mathrm{g}$., en el Barbate; $6.610 \mu \mathrm{g} \mathrm{Fe} / \mathrm{g}$., $27.841 \mu \mathrm{g} \mathrm{Cu} / \mathrm{g}$ en el Guadalquivir, datos referidos a peso seco).

En el tejido de musculatura dorsal de las lisas con peso $\leqslant 700 \mathrm{~g}$., las concentraciones medias de hierro, cobre, manganeso y cinc son en el estuario del Guadalquivir 1,$74 ; 2,30 ; 1,73$ y 1,27 veces respectivamente mayores que en el del Barbate, siendo signifi- 


\begin{tabular}{|c|c|c|c|c|c|c|c|}
\hline \multirow{2}{*}{\multicolumn{2}{|c|}{ n }} & \multicolumn{2}{|c|}{ Hígados } & \multicolumn{2}{|c|}{ Músculos } & \multicolumn{2}{|c|}{$\begin{array}{l}\text { Conc. hígados/ } \\
\text { Conc. músculos }\end{array}$} \\
\hline & & $\begin{array}{l}\text { Barbate } \\
25\end{array}$ & $\begin{array}{c}\text { Guadalquivir } \\
12\end{array}$ & $\begin{array}{c}\text { Barbate } \\
28\end{array}$ & $\begin{array}{c}\text { Guadalquivir } \\
21\end{array}$ & Barbate & Guadalquivir \\
\hline $\begin{array}{c}\mathrm{Fe} \\
\mu \mathrm{g} / \mathrm{g} \\
\text { peso seco }\end{array}$ & $\begin{array}{l}\text { Máx. } \\
\text { Mín. } \\
\vec{X} \\
\text { S }\end{array}$ & $\begin{array}{l}3.181 \\
445 \\
1.313(\mathrm{a}) \\
716,8\end{array}$ & $\begin{array}{l}3.820 \\
1.282 \\
2.277(\mathrm{a}) \\
737,3\end{array}$ & $\begin{array}{l}86,1 \\
12,5 \\
31,3(\mathrm{e})^{* * *} \\
16,8\end{array}$ & $\begin{array}{l}124 \\
16,5 \\
54,5(\mathrm{e}) \\
24,06\end{array}$ & 41,9 & 41,8 \\
\hline $\begin{array}{c}\mathrm{Cu} \\
\mu \mathrm{g} / \mathrm{g} \\
\text { peso seco }\end{array}$ & $\begin{array}{l}\text { Máx. } \\
\text { Mín. } \\
\text { X } \\
\text { s }\end{array}$ & $\begin{array}{l}1.078 \\
22,7 \\
275(\mathrm{~b})^{* \star *} \\
284,9\end{array}$ & $\begin{array}{l}19.444 \\
726 \\
6.234(\mathrm{~b}) \\
5.224\end{array}$ & $\begin{array}{c}19,5 \\
3,72 \\
6,78(f) \\
3,825\end{array}$ & $\begin{array}{l}17,1 \\
4,5 \\
8,55(\mathrm{f}) \\
3,701\end{array}$ & 40,6 & 729,1 \\
\hline $\begin{array}{c}\mathrm{Mn} \\
\mu \mathrm{g} / \mathrm{g} \\
\text { peso seco }\end{array}$ & $\begin{array}{l}\text { Max. } \\
\text { Min. } \\
\bar{x} \\
\text { s }\end{array}$ & $\begin{array}{l}47,2 \\
4,41 \\
14,4(\mathrm{c}) \\
10,81\end{array}$ & $\begin{array}{l}43.7 \\
1,7 \\
14.3(\mathrm{c}) \\
13,9\end{array}$ & $\begin{array}{l}4,08 \\
0,00 \\
0,95(\mathrm{~g}) \\
0,979\end{array}$ & $\begin{array}{l}3,85 \\
0,46 \\
1,64(\mathrm{~g})^{*} \\
0,915\end{array}$ & 15,2 & 8,7 \\
\hline $\begin{array}{c}\mathrm{Zn} \\
\mu \mathrm{g} / \mathrm{g} \\
\text { peso seco }\end{array}$ & $\begin{array}{l}\text { Máx. } \\
\text { Mín. } \\
\vec{X} \\
\text { s }\end{array}$ & $\begin{array}{l}307 \\
125 \\
205(d) \\
47,8\end{array}$ & $\begin{array}{l}588 \\
132 \\
321(\mathrm{~d}) \\
136,5\end{array}$ & $\begin{array}{l}50,0 \\
22,3 \\
31,8(\mathrm{~h})^{* *} \\
8,27\end{array}$ & $\begin{array}{l}71,5 \\
22,2 \\
40,5(h) \\
12,53\end{array}$ & 6,4 & 7,9 \\
\hline
\end{tabular}

Las letras iguales entre paréntesis, muestran los conjuntos de datos comparados entre sí por el criterio «t» de Student.

Grado de significación: * $\mathrm{p}<0,5$

$$
\begin{array}{rl}
* * * & p<0,01 \\
p & <0,001
\end{array}
$$

Tabla 3.-(Mugilauratus). Concentraciones de metales en hígados y tejido de musculatura dorsal de individuos con peso $<700 \mathrm{~g}$. y relación de las concentraciones medias hígados/músculos. (n, número de individuos; valores máximos y mínimos; $\overline{\mathbf{x}}$, valores medios; s, desviacionestípicas).

(Mugil auratus). Metal concentrations in livers and dorsal muscle tissues of specimens weiphing less than $700 \mathrm{~g}$, and liver/muscle mean concentration ratios. (n, number of specimens; Max., maximum values; Min., minimum values; $\bar{x}$, mean values; s, standard deviations).

cativas las diferencias encontradas en los casos del hierro $(P<0,001)$, manganeso $(P<0,5)$ y cinc $(\mathrm{P}<0,001)$. (Tabla 3).

\section{DISCUSION}

Los valores medios de las concentraciones de hierro, cobre y cinc en Crassostrea angulata encontrados en el presente estudio (Tabla 1), son algo mayores que los obtenidos por Establier $(1969,1977)$ y Establier y Pascual (1974) en las mismas zonas (Tabla 4). Estas diferencias pueden atribuirse a que estos autores estudiaron ostiones con tallas mayores que los del presente trabajo, ya que existe una relación directa entre el peso y la talla e inversa entre el peso y las concentraciones de hierro, cobre y cinc (Cordón,
1985). En cambio, no existe una relación clara entre la concentración de manganeso y el peso, por lo que los valores medios de este elemento en los individuos estudiados concuerdan con los de Establier (1969) y Establier y Pascual (1974) (Tabla 4).

El hecho más importante respecto a los ostiones es que los valores medios de cobre y cinc de los individuos del Guadalquivir son muy superiores a los del Barbate, resultados que concuerdan con los obtenidos por Establier $(1969,1977)$ y Establier y Pascual (1974) (Tablas 1 y 4).

Es interesante resaltar que todos los valores de concentración de cobre en los ostiones estudiados en el estuario del Guadalquivir, y por tanto su valor medio, $2.979 \mu \mathrm{g} / \mathrm{g}$ peso seco, están por encima del límite considerado como admisible para el consumo humano (150-200 $\mu \mathrm{g} \mathrm{Cu} / \mathrm{g}$ peso húmedo, equivalente a 
$\mathrm{Fe}$

1. Barbate
Guadalquivir
Barbate
2. Guadalquivir
3. Guadalquivir

4. Long Island Sound (EE.UU.)

5. Bahía de St. Louis (EE.UU.)
$\mathrm{Cu}$

Mn

Zn

$\mu \mathrm{g} / \mathrm{g}$ peso seco

Crassostrea angulata

$\begin{array}{crcr}283 & 251 & 47,5 & 3.547 \\ 328 & 1.868 & 42,9 & 7.687 \\ - & 167 & - & 2.444 \\ - & 1.643 & - & 13.760 \\ 303 & 1.287 & 52,0 & 5.702\end{array}$

Crassostrea virginica

$-\quad 574-1083$
$-\quad 46-197$

$262-5.131$

Crassostrea margaritacea

5-97 $\quad 0,8-17,6$

126-6.516
6. Costa sur de Suráfrica

53-380

I. Establier(1969);2. Establier(1977);Establier y Pascual (1974);4. Zaroogian (1979):5. Lytle y Lytle (1982);6. Watling y Watling (1974).

Tabla 4.-Concentraciones de $\mathrm{Fe}, \mathrm{Cu}, \mathrm{Mn}, \mathrm{y} \mathrm{Zn}$ en Crassostrea angulata, Crassostrea virginica y Crassostrea margaritacea. Table 4.- Concentrationsof $\mathrm{Fe}, \mathrm{Cu}, \mathrm{Mn}$ and $\mathrm{Zn}$ in Crassostrea angulata, Crassostrea virginica y Crassostrea margaritacea.

unos $1.000 \mu \mathrm{g} \mathrm{Cu} / \mathrm{g}$ peso seco) y que sólo uno de los 37 individuos analizados en el estuario del Barbate $(1.520 \mu \mathrm{g} \mathrm{Cu} / \mathrm{g}$.) supera ese límite.

En comparación con otros estudios realizados en ostras, las concentraciones de cobre encontradas en C. angulata en el estuario del Barbate, son del mismo orden que las halladas por Zaroogian (1979), y mucho mayores que las encontradas por Lytle y Lytle (1982) en Crassostrea virginica (Tabla 4). También son mayores las concentraciones oe cinc de todos los ostiones analizados en el presente estudio, respecto a los valores encontrados por estos últimos autores. Por otra parte las concentraciones de hierro, cobre, manganeso y cinc encontradas por Watling y Watling (1983) en Crassostrea margaritacea. son inferiores que los de C. angulata tanto del Barbate como del Guadalquivir (Tabla 4).

De la comparación de las Tablas 1 y 2 se desprende que salvo algún resultado correspondiente a los músculos de las lubinas. todas las concentraciones de metales en los hígados y músculos de lisas o lubinas son mayores en los especimenes capturados en el Guadalquivir. También puede afirmarse que con una posible excepción todas las concentraciones de metales encontradas en los hígados y los músculos de las lisas son mayores que las correspondientes de las lubinas.

La observación de los aparatos digestivos realizada durante la disección, mostró la presencia de abundan- tes restos de sedimentos en las lisas, mientras que las lubinas contenían casi exclusivamente crustáceos y peces de pequeño tamaño perfectamente identificables. Dado que los metales pesados se concentran en los sedimentos (Turekian, 1977) y la mayor contaminación a este respecto de los del estuario del Guadalquivir (Cordón, 1985), los resultados generales expuestos parecen totalmente explicables.

Particularizando, pueden ponerse de relieve los siguientes extremos: a) al ser los hígados órganos acumuladores (Honda et al., 1983), en ellos se manifiesta más acusadamente las diferencias atribuibles a la contaminación del medio y al régimen alimentario de estas dos especies. Destaca a este respecto la acumulación de cobre en los hígados de las lisas del Guadalquivir cuya relación de concentraciones medias hígado/músculo es 729 frente a 40,6 para las del Barbate; b) al ser la contaminación por cobre muy elevada en los individuos de las dos especies capturados en el Guadalquivir, se pone de relieve que este metal está presente en las dietas alimentarias de ambas especies, las cuales tienen la facultad de acumularlo; y c) esta circunstancia no se da para el cinc y el hierro que sólo son asequibles, o sólo se acumulan en grandes cantidades en las lisas.

Según Uysal y Tuncer (1982), concentraciones de $23.3 \mu \mathrm{g} \mathrm{Fe} / \mathrm{g}, 2,15 \mu \mathrm{g} \mathrm{Cu} / \mathrm{g}, 3,05 \mu \mathrm{g} \mathrm{Mn} / \mathrm{ga}$ y $24,03 \mu \mathrm{g}$ $\mathrm{Zn} / \mathrm{g}$, y $42.9 \mu \mathrm{g} \mathrm{Fe} / \mathrm{g}, 3,85 \mu \mathrm{g} \mathrm{Cu} / \mathrm{g}, 1.96 \mu \mathrm{g} \mathrm{Mn} / \mathrm{g}$ y $17,94 \mu \mathrm{g} \mathrm{Zn} / \mathrm{g}$. (referido a peso seco), en tejido comes- 
tible de Dicentrarchus labrax y Mugil spp., respectivamente, capturados en la bahía de Izmir en Turquía, no son altamente tóxicas para el consumo humano. De los valores encontrados en el presente trabajo para músculo, sólo los de cobre en ambas especies y los de cinc en lisas son mayores que los anteriores, aunque sus niveles no son suficientemente altos como para entrañar riesgo para la salud del consumidor. Los de manganeso, en cambio, son menores en todos los individuos analizados en este trabajo.

\section{CONCLUSIONES}

Los resultados expuestos ponen de relieve la existencia en el estuario del Guadalquivir de una fuente de contaminación metálica más acusada que en el del Barbate. La manifestación de dicha contaminación se aprecia más fácilmente en el cobre de los ostiones y de los hígados de lubinas y lisas, aunque también se manifiesta en el cinc de los ostiones y en el hierro y cinc de los hígados de lisas.

\section{BIBLIOGRAFIA}

Cordón, R. 1985. Estudio comparativo de la contaminación por metales pesados en aguas, sedimentos y animales de los estuarios de los ríos Guadalquivir y Barbate. Datos sin publicar.
Establier, R. 1969. Contenido en cobre, hierro, manganeso y cinc de los ostiones (Crassostrea angulata) de las costas de Cádiz. Inv. Pesq. 33: 335-343.

Establier, R. 1977. Estudio de la contaminación marina por metales pesados y sus efectos biológicos. Inf. Técn. Inst. Inv Pesq. 47: 3-36.

Establier, R. y Pascual, E. 1974. Estudios del cobre, hierro, manganeso y cinc en ostiones (Crassostrea angulata) del Golfo de Cadiz. Inv. Pesq. 38: 371-384.

Honda, K.; Sahrul, M. y Hidaka, H. 1983. Organ and tissue distribution of heavy metals, and their growth-related changes in Antartic fish Pagothenia borchgrevinki Agric. Biol. Chem. 47: 2.521-2.532.

Katz, S.A.; Jenniss, S.W.; Tout, R.E. y Chatt, A. 1981. Comparison of sample preparation methods for the determination of metals in sewage sludges by flame atomic absorption spectrometry. Intern. J. Environ. Anal. Chem. 9: 209-220.

Litle, T.F. y Lytle, J.S. 1982. Heavy metals in oysters and clams of St. Louis Bay, Mississippi. Bull. Environ. Contam. Toxicol. 29: 50-57.

Ritter, C.J.; Bergman, S.C.; Cothern, C.R. y Zamierowski, E.E. 1978. Comparison os sample preparatin techniques for atomic absorption analysis of sewage sludge and soil. $A t . A b-$ sorpt. Newls. 17: 70-72.

Turekian, K.K. 1977. The fate of metals in the oceans. \&ochim. Consmochin. Acta. 41: 1.139-1.144.

Uysal, H. y Tuncer, S. 1981. Levels of heavy metals in some commercial food species in the Bay of Izmir (Turkey). VI Journées Etud. Pollutions. Cannes, C.I.E.S.M. 323-327. 